\title{
Efficacy of Local Treatments for Hepatocellular Carcinoma Involving the Inferior Vena Cava and/ or Right Atrium
}

This article was published in the following Dove Press journal: Journal of Hepatocellular Carcinoma

\author{
Han Ah Lee (D) \\ Chai Hong Rim (iD) ${ }^{2}$ \\ 'Department of Internal Medicine, Korea \\ University Anam Hospital, Korea \\ University Medical College, Seoul, \\ Republic of Korea; ${ }^{2}$ Department of \\ Radiation Oncology, Korea University \\ Ansan Hospital, Korea University Medical \\ College, Ansan, Gyeonggido, Republic of \\ Korea
}

Correspondence: Chai Hong Rim Department of Radiation Oncology, Korea University Ansan Hospital, 123 JeokgeumRo, Danwon-Gu, Ansan, Gyeonggido I5355, Republic of Korea

Tel +82 3I 4I26850

Fax +82314126851

Email crusion3@naver.com

\begin{abstract}
Major vessel invasion is frequently observed in locally advanced hepatocellular carcinoma (HCC). The most commonly involved major vessel is the portal vein; however, approximately $4 \%$ of patients with HCC have inferior vena cava (IVC) and/or right atrium (RA) invasion. Although these conditions have dismal prognoses, local treatments have not been regularly administered because they may affect systemic circulation. Owing to recent technological advances, various local treatments including surgery and external radiotherapy have increasingly been performed in these patients. Since irradiating tumorous lesions in the liver while preserving normal tissues is possible and major vessels are relatively resistant to radiation, external radiotherapy has been a feasible palliative modality for treating vessel-invasive HCC. In addition, systemic fatal complications that were initially a cause of concern have become rare after radiotherapy. While invasive procedures such as extracorporeal circulation or hepatic vascular exclusion may be required, pioneering surgeons have performed surgical resections in selected patients and have obtained promising results. Surgery has shown the best survival outcomes compared to other treatment options including radiotherapy, but the possibility of perioperative morbidity should be considered. In addition, a combination of local treatment and novel systemic agents, which demonstrated better survival and response rates than sorafenib, is expected to maximize therapeutic effects. In this review, we have discussed the most recent treatments for HCC with IVC and/or RA involvement and have provided information intended to guide therapeutic decisions and facilitate future research.
\end{abstract}

Keywords: hepatocellular carcinoma, inferior vena cava, right atrium, surgery, radiotherapy

\section{Introduction}

The prevalence of hepatocellular carcinoma (HCC) varies across geographical regions; its incidence is especially high in East Asia, whereas it is relatively rare in Western countries. ${ }^{1}$ Furthermore, the causes and characteristics of HCC also differ significantly between regions. HCC in Western countries is mainly caused by alcoholic hepatitis, non-alcoholic fatty liver disease, and hepatitis $\mathrm{C}$ infection. Patients with HCC in these countries tend to have advanced cirrhosis or impaired liver function at the time of diagnosis of their malignancy. In contrast, the major cause of liver cancer in East Asia is the hepatitis B virus; patients with HCC in this region tend to have preserved liver function, but they are often diagnosed with locally advanced tumors. ${ }^{2,3}$

Locally advanced HCC tumors have a tendency to invade the major vessels of the liver. ${ }^{4}$ In a nationwide analysis based on data from the Korean Liver Cancer 
Study Group, one-third of all patients with HCC were categorized as having Barcelona-Clinic Liver Cancer (BCLC) stage $\mathrm{C}$ HCC, and approximately $60 \%$ were diagnosed with major vessel invasion. ${ }^{5}$ The most common form of vessel invasion is portal vein thrombosis (PVT). Previously, HCC with PVT was considered to have a dismal prognosis, with limited treatment options available. However, oncologic outcomes have been improving owing to the availability of local treatments including external beam radiotherapy (EBRT), internal radiotherapy, surgery, and systemic agents, which have demonstrated their benefits in recent decades. ${ }^{6-9}$

Invasion of the inferior vena cava (IVC) and/or right atrium (RA) is a much rarer type of vessel invasion. In a study by Lee et $\mathrm{al}^{10}$, approximately $4 \%$ of all HCC patients had IVC and/or RA invasion. Due to its rarity, its clinical outcomes have not been collectively studied, but have only been described in case reports and small case series. In addition, there have been concerns that administration of local treatment to patients with IVC and/or RA thrombosis might result in serious systemic complications such as pulmonary embolism or cardiac failure. However, EBRT has been shown to be feasible and efficient in recent studies, ${ }^{6,11}$ and pioneering surgeons have reported promising outcomes. ${ }^{12,13}$

In this review, we have discussed the efficacy and feasibility of local treatments including EBRT and surgery, as well as future therapeutic strategies that can be considered alongside systemic treatments.

\section{External Beam Radiotherapy}

Although EBRT has not been accepted as a standard modality for HCC owing to a lack of evidence from Phase 3 trials, it has been commonly used for palliation in patients with vessel invasion. In these patients, local modalities such as ablation and resection have been contraindicated owing to the risk of bleeding; however, EBRT remains relatively feasible. For example, the doses of EBRT in a recent series of patients with PVT mostly ranged from 45 to $60 \mathrm{~Gy}$, which yielded pooled response rates of $50-70 \%$ and local control rates of approximately $\geq 83 \%$. ${ }^{6}$ The tolerated radiation dose for major vessels, which has been commonly extrapolated from stereotactic body radiotherapy (SBRT) studies of lung cancer, exceeds 100 Gy prescribed in 2 Gy fractions. ${ }^{6}$ Therefore, thrombosis in the IVC and/or RA has also been a candidate for palliative EBRT in clinical practice, with meaningful local palliation expected without damage to major vessels.
Two important pioneering studies of EBRT for HCC with IVC and/or RA thrombosis were performed at tertiary centers in China and Korea. Zeng et $\mathrm{al}^{14}$ reported the treatment outcomes of 158 patients with $\mathrm{HCC}$ who had portal vein (114 patients) and/or IVC/RA thrombosis (44 patients) recruited between 1998 and 2003 at Zhongshan Hospital, Fudan University. The median survival in patients who underwent EBRT was 8 months, whereas that in non-EBRT-treated patients was 4 months. Among the 44 patients with IVC and/or RA thrombosis, 14 were treated with EBRT. Patients with IVC/RA thrombosis who did not undergo EBRT had poorer survival than their counterparts with PVT (median survival: 2 months, risk ratio: $3.14, \mathrm{p}<0.001$ ), whereas longer survival was achieved in patients who received EBRT (median survival: 22 months, risk ratio: $0.208, \mathrm{p}=0.047$ ). The overall response rate after EBRT was $78.6 \%$, and no serious cardiopulmonary complications were reported. The study by Koo et $\mathrm{al}^{15}$ performed at Asan Medical Center, Korea, which is one of the largest studies to date, investigated $71 \mathrm{HCC}$ patients exhibiting IVC and/or RA thrombosis. Among them, 42 patients underwent combined treatment including transarterial chemoembolization (TACE) and EBRT, while 29 historical controls were treated with TACE alone. Combined TACE and EBRT showed better overall survival (median: 11.7 vs 4.7 months, $\mathrm{p}<0.01)$ and response rates $(42.9 \%$ vs $13.8 \%, p=0.01)$ than TACE alone. No grade $\geq 3$ or serious systemic complications were reported in the combined treatment arm.

Despite the feasibility of EBRT, concerns over systemic failure due to dislodged IVC or RA thrombus fragments have hindered its adoption. Rim et $\mathrm{al}^{6}$ performed a meta-analysis of case series that described HCC patients with IVC and/or RA thrombosis who underwent EBRT. The pooled 1- and 2-year overall survival rates were $53.6 \%(95 \%$ confidence interval [CI]: 45.7-61.3\%) and $36.9 \%$ (95\% CI: $27.2-42.4 \%$ ), respectively, while the response and local control rates were $59.2 \%$ (95\% CI: $39.0-76.7 \%$ ) and $83.8 \%$ (95\% CI: 78.8-97.1\%), respectively. Only 1 patient among the 164 patients experienced pulmonary embolism, and the overall grade $\geq 3$ complication rate was $1.2 \%$ ( 2 of 164 patients). This meta-analysis not only demonstrated the potent local controllability of tumors using EBRT but also significantly alleviated unfounded clinical concerns of fatal systemic complications due to "dislodged thrombi fragments." 
While the above studies were performed before the sorafenib era in $2008,{ }^{16}$ several others have reported more recent outcomes. Rim et $\mathrm{al}^{11}$ performed a multiinstitutional study registered as the Korean Radiation Oncology Group trial (KROG 17-10), to investigate the use of EBRT for HCC patients with IVC and/or RA thrombosis. Forty-nine patients who underwent EBRT during 2003-2017 at 6 institutions were recruited; the median survival was 10.1 months, while the 1- and 2-year survival rates were $43.5 \%$ and $30.1 \%$, respectively. Lou et $\mathrm{al}^{17}$ reported the outcomes from 3 Chinese institutions, including $75 \mathrm{HCC}$ patients exhibiting IVC and/or RA thrombosis who underwent EBRT between 2008 and 2016. The median survival was 10 months, while the 1- and 2-year survival rates were $38.7 \%$, and $13.3 \%$, respectively. In both studies, tumor multiplicity and high alphafetoprotein levels were significant factors for survival, which suggested the biological aggressiveness of the malignancy; in contrast, thrombi location (IVC only vs RA and/or IVC) was not a clinically meaningful factor.

Data from selected studies of EBRT in HCC patients with IVC and/or RA thrombosis are summarized in Table 1. Although EBRT was effective for local palliation, the overall survival rates were not satisfactory, as the median survival reported in these studies barely reached 1 year. Complications were generally mild, and pulmonary embolism caused by dislodged thrombi fragments was very rare. There appears to be no significant difference in oncologic outcomes between studies of patients recruited before and after 2008; this may be due to the highly heterogeneous clinical characteristics and advanced disease profiles (eg, lymphatic or extrahepatic metastasis, and coexisting PVT) of patients included in these studies.

\section{Surgical Resection}

HCCs with IVC and/or RA invasion are generally presumed to have poor prognoses, with expected survival of $<3$ months, while those with PVT have even poorer prognoses. ${ }^{14,18}$ In addition, given the possibility of sudden death due to circulatory failure, ${ }^{19}$ physicians have been highly cautious about performing surgeries that may burden the circulatory system. In HCCs with IVC thrombosis, total hepatic vascular exclusion is required to block IVC blood flow and perform thrombectomy. ${ }^{12}$ In cases of thrombus extension to the RA, extracorporeal circulation must be used for successful surgery. ${ }^{12,13}$ As such invasive surgical procedures are necessary, surgery has not been actively performed in these patients.
With advances in surgical techniques, resection and reconstruction of the IVC has been shown to be safe and effective for various malignant tumors, including renal cell carcinoma. ${ }^{20}$ In HCC, most previous studies were small case series or case reports; however, some pioneering surgeons have recently reported meaningful clinical experiences. The study by Matsukuma et $\mathrm{al}^{21}$ included 37 HCC patients with IVC and/or RA thrombosis treated at 3 hospitals in Osaka and Yamaguchi. The median survival was 13.8 months, and the recurrence-free survival was 5.2 months after curative resection. Complete resection was achieved in $67.6 \%$ of patients; those with postoperative residual liver disease had significantly poorer survival than those who did not (median survival: $8.3 \mathrm{vs}$ 18.7 months, $\mathrm{p}=0.009$ ). The grade $\geq 3$ complication rate was $17.9 \%$, including 2 patients with pulmonary embolisms, 2 patients with bile leaks, 1 patient with surgical infection, 1 patient with ascites, and 1 patient with bowel obstruction. Two patients died within 90 days of surgery. Furthermore, survival outcomes were similar between patients treated during 1997-2008 and those treated during 2009-2017 (ie, between the pre- and post-sorafenib era). Kasai et $\mathrm{al}^{22}$ reported the outcomes of 39 patients who underwent surgery; the median survival was 15.2 months, and the recurrence-free survival was 5.3 months after R0/1 resection (R0/1 resection rate: 79\%). Extracorporeal circulation use and extrahepatic metastases were significantly related to poor survival, while undergoing preoperative hepatic arterial infusion chemotherapy was associated with prolonged survival. Grade $\geq 3$ complications (mostly pleural effusion and ascites) occurred in $67 \%$ of patients, and the 30 -day postoperative mortality rate was $5 \%$.

\section{Comparison of Surgery and EBRT}

Some researchers compared the outcomes of primary surgery and EBRT. Li et al ${ }^{23}$ reported their clinical experience with $108 \mathrm{HCC}$ patients with IVC and/or RA thrombosis treated at the Eastern Hepatobiliary Surgery Center, 57 of whom received EBRT and 51 underwent surgery. Clinical characteristics of the 2 groups were not significantly different, except that the EBRT arm included a higher frequency of PVT with borderline statistical significance ( $24.6 \%$ vs $11.8 \%, \mathrm{p}=0.087$ ). The median survival, 1 -year survival rate, and 2-year survival rate were 12.8 months, $57.9 \%$, and $24.6 \%$, respectively, in the EBRT arm, and were 14.5 months, $58.8 \%$, and $21.6 \%$, respectively, in the surgery arm $(\mathrm{p}=0.466)$. Two $(3.9 \%)$ patients had grade $\geq 3$ complications in the surgery arm, while the EBRT arm had 


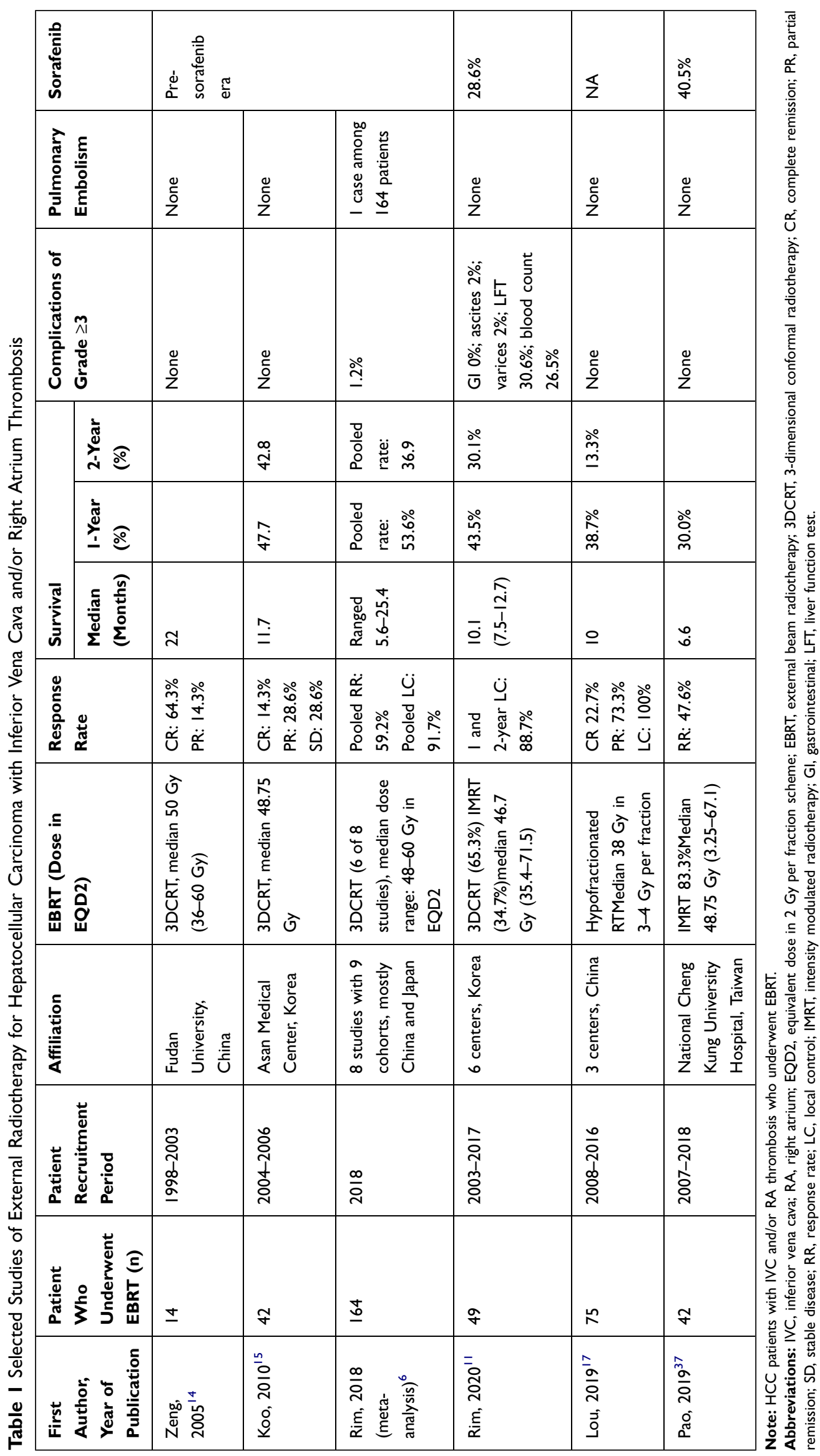


none. No serious complications such as early perioperative mortality, cardiac arrest, and radiation-induced liver disease occurred. Komatsu et $\mathrm{al}^{24}$ performed a matched cohort study of patients undergoing proton radiotherapy and those undergoing surgery at Kobe University, Japan; each arm had 19 patients with similar characteristics, except that there was a higher proportion of patients with Child-Pugh class A in the surgery arm, with borderline significance $(89.5 \%$ vs $57.9 \%, \mathrm{p}=0.058)$. The 1 -year and 3 -year survival rates were $68 \%$ and $25 \%$, respectively, in the proton therapy group, and were $34 \%$ and $14 \%$, respectively, in the surgery group ( $\mathrm{p}=0.106)$. Grade $\geq 3$ complications were not reported in the proton therapy group but were reported in $5(26.3 \%)$ patients in the surgery group, including 1 fatality.

Our team has previously published a clinical metaanalysis of EBRT studies on HCC patients with IVC and/ or RA thromboses. ${ }^{11}$ We also recently performed an updated meta-analysis of 18 studies involving 22 cohorts comparing EBRT with surgery. ${ }^{25}$ Each cohort included at least 10 relevant patients, with the entire study including 755 patients. The surgery arm had a significantly higher proportion of patients with Child-Pugh class A (pooled rate: $90.1 \%$ vs $69.1 \%, p=0.005)$ and a lower rate of extrahepatic metastasis (pooled rate: $14.5 \%$ vs $34.5 \%, \mathrm{p}=0.067$ ) than the EBRT arm, with borderline significance. The pooled median survival period was 15.3 and 11.7 months in the surgery and EBRT arms, respectively. The 1-year survival rates were $62.4 \%$ (95\% CI: $53.8-70.3$ ) and $48.8 \%$ (95\% CI: 40.9-56.8) in the surgery and EBRT arms, respectively $(\mathrm{p}=0.023)$, while the corresponding 2-year survival rates were $26.9 \%$ (95\% CI: 20.7-34.2) and $27.5 \% \quad(95 \%$ CI: 19.7-37.1), respectively $(\mathrm{p}=0.913)$. Grade $\geq 3$ complications were rarely reported in the EBRT arm, but their incidence varied between 3.9-67\% in the surgery arm.

Selected studies concerning surgery and those comparing surgery and EBRT are summarized in Table 2. In most of the studies regarding surgery, although previously contraindicated, median survival times were longer than 1 year; the 1-year survival rate was approximately $60 \%$. Although survival rates after surgery appeared to be somewhat higher than those after EBRT, patients who underwent surgery may have had relatively more favorable clinical characteristics. In addition, grade $\geq 3$ complications were more frequent after surgery, and although rare, the possibility of perioperative mortality cannot be ignored. In conclusion, surgery could be the most effective local modality for achieving the best survival benefit. In patients who are not indicated to undergo surgery, EBRT could be a valid and less invasive option with potent palliative efficacy.

\section{Transarterial Chemoembolization}

TACE has been the most commonly administered local treatment modality for $\mathrm{HCC}$ in recent decades and is the recommended standard treatment for intermediate-stage HCC. $^{26}$ TACE alone is not generally indicated for HCC with IVC and RA thrombosis, owing to poor outcomes and difficulty in achieving sustained local control. ${ }^{27,28}$ In addition to administration as a definitive local modality, TACE has been administered before or after treatment with surgery or EBRT. ${ }^{11,12,15,17}$

Few publications have described TACE as a primary treatment. Chern et $\mathrm{al}^{27}$ reported the outcomes of $26 \mathrm{HCC}$ patients with IVC and/or RA thrombosis; the median survival was 4.2 months and the 1-year and 2-year survival rates were $41 \%$ and $25 \%$, respectively. The overall response rate was $53.8 \%$, and the median survival of responders was significantly longer than that of nonresponders ( 13.5 vs 3.3 months, $\mathrm{p}<0.002)$. Transient deterioration of liver function $(100 \%)$, abdominal pain $(70.9 \%)$, vomiting $(61.5 \%)$, and fever $(80.5 \%)$ were common complications. Critical pulmonary embolisms were not observed. In the abovementioned EBRT study by Koo et $\mathrm{al}^{15}$, patients in the TACE-only arm (the historical controls) were compared with those in the TACE plus EBRT arm; baseline clinical profiles were well matched, except that tumor sizes in the TACE-only arm were relatively larger than those in the TACE plus EBRT arm (mean primary tumor size: $12.9 \mathrm{~cm}$ vs $10.0 \mathrm{~cm}, \mathrm{p}<0.01$ ). The overall survival and response rate were better in the TACE plus EBRT arm than in the TACE-only arm (median survival: 11.7 vs 4.7 months, $\mathrm{p}<0.01$; response rate: $42.8 \%$ vs $13.8 \%, \mathrm{p}=0.01$ ). Radiation-induced liver disease or grade $\geq 3$ complications due to EBRT were not reported. Wang et $\mathrm{al}^{12}$ performed a comparative study of $56 \mathrm{HCC}$ patients with IVC and/or RA thrombosis who were categorized into 3 treatment subgroups - surgery (25 patients), TACE (20 patients), and no treatment (11 patients). The clinical characteristics of patients in all arms were not significantly different, except that the surgery arm had a higher proportion of patients with elevated alpha-fetoprotein levels. The median survival in the surgery, TACE, and no treatment groups were $19,4.5$, and 5 months, respectively. Two (8\%) 


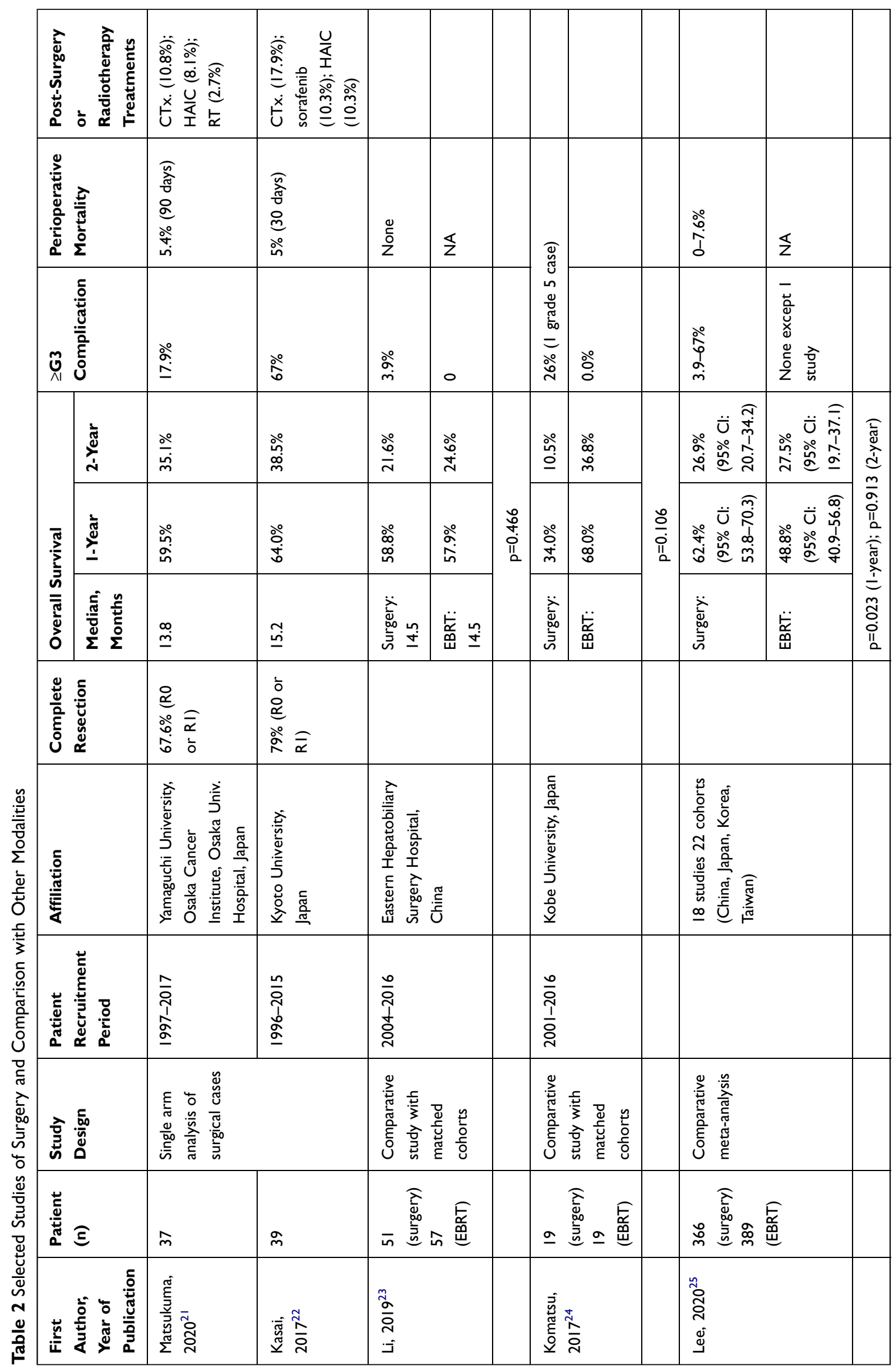




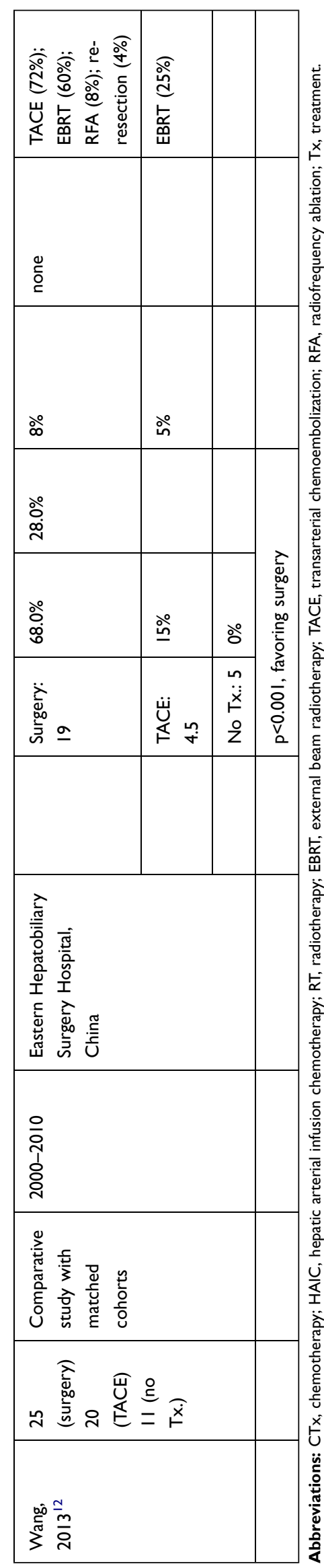

patients in the surgery arm experienced grade 3 complications.

To summarize, the primary application of TACE appears to have a lower oncologic efficacy than EBRT or surgery. TACE can be considered an adjuvant or salvage modality rather than a definitive one, in which the patient's clinical condition and risk of post-embolization syndrome ought to be considered.

\section{Pattern of Recurrence and Role of Systemic Treatment}

Despite the improved response rates and survival outcomes of HCC patients with IVC and/or RA invasion with the active and widespread use of local treatments, progression rates after effective local treatment remain high. Few studies have evaluated HCC progression after locoregional treatment in such patients. A recent study found that the median progression-free survival was 4 months after EBRT $^{6}$ and 5.2-5.3 months after surgery. ${ }^{21,22,29,30}$ Furthermore, the progression-free rate at 3-4 months after TACE was $37.9 \% .{ }^{15}$ Considering the short duration taken for HCC progression, remaining microscopic or macroscopic metastases (rather than multicentric carcinogenesis) would provoke cancer progression after local control, which characteristically occurs within 2 years after treatment. ${ }^{31}$

The first sites of progression in such patients after surgical resection are the liver (23.1-69.2\%) and lungs (13.5-53.8\%). ${ }^{13,21,22,29}$ Although exact reasons for the high prevalence of lung metastases after surgical resection is unknown, it is possible that lung micrometastases could already be present before resection, or that the procedure itself could disseminate tumor cells systemically through the IVC. ${ }^{32,33}$ After EBRT, the most common sites of first progression were reported to be the liver (21.5-61.5\%), followed by the lung (13.5-42.9\%). ${ }^{11,34}$ There have been increased concerns of pulmonary metastasis in IVC/RAinvasive $\mathrm{HCC}$, due to ability of a RA tumor thrombus to flow into the pulmonary vessels. ${ }^{35}$ It has further been suggested that RT could destabilize an IVC and/or RA thrombus, leading to the hematogenous dissemination of cancer cells, although epidemiologic data supporting these concerns are insufficient. ${ }^{11,34}$ Furthermore, it is difficult to determine whether such newly developed lung metastases originated from thrombus dislodgment during RT, or reflect the natural progression of the disease given that 
the lung is one of the most frequent locations of distant metastases in $\mathrm{HCC}^{36}$

Clearly, local control alone appears to be insufficient for preventing further progression in a substantial proportion of HCC patients with IVC/RA invasion. Concurrent or adjuvant systemic therapy has been considered to prevent further progression; however, only few studies have evaluated the effects of systemic therapy in these patients. Rim et al reported longer median overall survival in patients who received concurrent or additional systemic treatment than in those who did not (12.2 vs 8.4 months), but the difference was not significant $(\mathrm{p}=0.054) .{ }^{11}$ According to the study by Pao et al, systemic therapy with RT was associated with longer median lung metastasis-free survival (5.9 vs 2.1 months, $\mathrm{p}=0.0033$ ) in $\mathrm{HCC}$ patients with IVC and/or RA involvement. ${ }^{34}$ Among patients with BCLC stage $\mathrm{C}$ HCC, the recurrence rate after curative resection was markedly lower in those treated with adjuvant sorafenib therapy than in those who were not $(44.1 \%$ vs $75.0 \%$, $\mathrm{p}=0.002) .{ }^{37}$ Furthermore, Liu et al performed a study involving patients with BCLC stage $\mathrm{C}$ HCC and Child-Pugh class A, and reported a significantly longer time to progression (29 months vs 22 months, $\mathrm{p}=0.0 .41$ ) and median overall survival (37 months vs 30 months, $\mathrm{p}=0.01$ ) in patients treated with sorafenib after resection than in those who only underwent resection. $^{38}$

Based on previous data, the combination of local primary tumor control and systemic microscopic metastasis control could yield better treatment outcomes in HCC patients with IVC and/or RA invasion. ${ }^{39,40}$ A randomized Phase III study is currently recruiting advanced-stage HCC patients to investigate the effects of SBRT with or without sorafenib (NCT01730937). Another randomized Phase II trial will recruit patients with portal vein invasion and compare the efficacy of surgery plus sorafenib and sorafenib alone (NCT03971201). In addition, the role of lenvatinib (the alternative first-line systemic agent to sorafenib) as an adjuvant therapy for these patients is expected, because it has shown superior objective response rates and progression-free survival compared to sorafenib. ${ }^{41}$ However, as the treatment efficacy of lenvatinib in patients with major vessel invasion has not been fully evaluated, there is keen interest in upcoming data. ${ }^{39}$ In line with this, a randomized phase III study is currently recruiting patients with BCLC stage $\mathrm{B}$ or C HCC to investigate the efficacy of lenvatinib after resection (NCT04227808).
Recently, immunotherapy has been introduced to treat HCC; however, many immunosuppressive mechanisms limit the effectiveness of immune-based monotherapy. The combination of RT and immune checkpoint inhibitors (ICIs) has gained extensive attention because RT has proimmunogenic properties. $^{40,42}$ Preclinical studies have shown that RT promotes systemic immune responses by enhancing immunogenic antigen presentation, proinflammatory cytokines stimulation, and increasing PD-L1 expression. ${ }^{43-47}$ Furthermore, a combination of ICI and RT could be helpful in overcoming radiation-induced immunosuppressive effects that lead to radioresistance. ${ }^{48}$ The benefits of combined ICI and RT have been reported in patients with other malignancies; ${ }^{49,50}$ however, only 2 preclinical studies have evaluated the mechanism and efficacy of ICIs and RT in murine HCC models. ${ }^{51,52}$ Currently, a phase II trial is recruiting advanced-stage HCC patients treated with pembrolizumab and SBRT (NCT03316872), and another Phase I study will be evaluating the treatment outcomes of SBRT followed by nivolumab or ipilimumab therapy in patients with unresectable HCC (NCT03203304).

Adjuvant immune-based therapies after surgical resection of $\mathrm{HCC}$ have been introduced. Lee et $\mathrm{al}^{53}$ evaluated the efficacy of cytokine-induced killer cells in the adjuvant setting of patients with resected HCC, and found that this therapy was significantly associated with improved overall and recurrence-free survival. As previously published data are insufficient, questions remain regarding the safety and efficacy of adjuvant immunotherapies in advanced-stage HCC patients undergoing surgery. There may be promise in the currently ongoing phase III study testing nivolumab in the adjuvant setting for resectable HCC regardless of disease stage (NCT03383458, CheckMate 9DX). In addition, the potent combination therapy of atezolizumab and bevacizumab is being investigated for its efficacy as an adjuvant therapy for patients with resectable HCC (NCT04102098, IMbrave050). As more immunotherapies prove to be effective against advanced-stage $\mathrm{HCC}$, recent results could presumably translate into achieving successful combinations of local control and adjuvant immunotherapy for selected patients. Ongoing trials of systemic agents in the adjuvant setting after locoregional treatment of advanced-stage HCC are listed in Table 3.

\section{Concluding Remarks}

There has been reluctance to administer local treatment for HCC patients with IVC and/or RA involvement as it might 


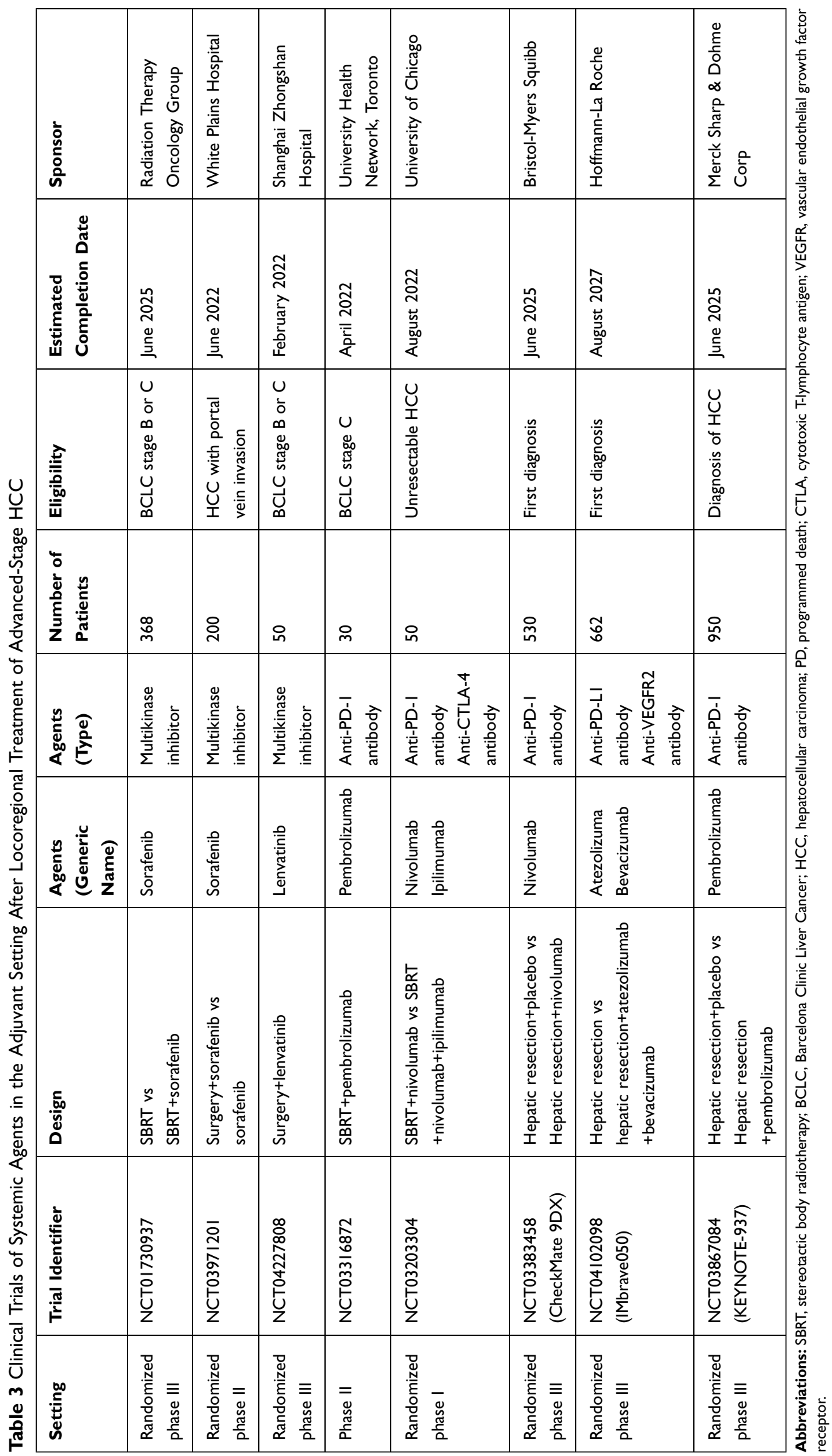


directly affect the circulatory system. As the incidence of such HCC type is relatively low, local treatment has not been a major subject of interest for therapeutic research. However, recent studies have shown that surgery is an effective local treatment and provides superior survival benefits for these patients. Efforts to minimize morbidity and careful patient selection are required. Non-invasive EBRT could be another useful treatment option, as it is effective in improving oncologic outcomes, and fatal respiratory complications that were originally of concern rarely occur. Major limitations of current treatments include the high recurrence rate and short progressionfree survival despite aggressive treatment attempts. Although recent studies have shown improved survival after local treatment, other important clinical outcomes including quality of life or post-treatment complication rates should be investigated in future studies. As newer systemic agents have shown higher response rates than sorafenib, their use is expected to further reduce recurrence rates after local control. In particular, adjuvant or combined immunotherapy, which has recently shown promising results, may enhance the prognosis of $\mathrm{HCC}$ with IVC and/or RA involvement in upcoming studies.

\section{Abbreviations}

BCLC, Barcelona Clinic Liver Cancer; CI, confidence interval; EBRT, external beam radiotherapy; HCC, hepatocellular carcinoma; ICI, immune checkpoint inhibitor; IVC, inferior vena cava; PVT, portal vein invasion; RA, right atrium; RT, radiotherapy; SBRT, stereotactic body radiotherapy; TACE, transarterial chemoembolization.

\section{Ethical Considerations}

Ethical approval was not required because this study retrieved and synthesized data from papers that are already published.

\section{Acknowledgments}

This study was supported by the National Research Fund of Korea (NRF-2019M2D2A1A01031560). The research grant supported only methodological aspects including statistical analysis and linguistic correction, and did not influence major contents including the results and conclusions.

\section{Author Contributions}

All authors contributed to data analysis, drafting and revising of the article, agreed on the journal to which the article will be submitted, gave final approval of the version to be published, and agree to be accountable for all aspects of the work.

\section{Disclosure}

None of the authors have any conflicts of interest to disclose.

\section{References}

1. Bray F, Ferlay J, Soerjomataram I, Siegel RL, Torre LA, Jemal A. Global cancer statistics 2018: GLOBOCAN estimates of incidence and mortality worldwide for 36 cancers in 185 countries. CA Cancer J Clin. 2018;68:394-424.

2. Choo SP, Tan WL, Goh BK, Tai WM, Zhu AX. Comparison of hepatocellular carcinoma in Eastern versus Western populations. Cancer. 2016;122:3430-3446.

3. Sinn DH, Gwak G-Y, Cho J, Paik SW, Yoo BC, Roques P. Comparison of clinical manifestations and outcomes between hepatitis $\mathrm{B}$ virus-and hepatitis $\mathrm{C}$ virus-related hepatocellular carcinoma: analysis of a nationwide cohort. PLoS One. 2014;9:e112184. doi:10.1371/journal.pone. 0112184

4. Llovet JM, Bustamante J, Castells A, et al. Natural history of untreated nonsurgical hepatocellular carcinoma: rationale for the design and evaluation of therapeutic trials. Hepatology. 1999;29:62-67. doi:10.1002/hep.510290145

5. Lee J, Yoon WS, Koom WS, Rim CH. Role of local treatment including radiotherapy in Barcelona Clinic of Liver Cancer stage C patients: a nationwide cohort analysis in South Korea. Cancer Manag Res. 2019;11:1373-1382. doi:10.2147/CMAR.S193761

6. Rim CH, Kim CY, Yang DS, Yoon WS. Comparison of radiation therapy modalities for hepatocellular carcinoma with portal vein thrombosis: a meta-analysis and systematic review. Radiother Oncol. 2018;129:112-122. doi:10.1016/j.radonc.2017.11.013

7. Rim $\mathrm{CH}$, Yoon WS. Leaflet manual of external beam radiation therapy for hepatocellular carcinoma: a review of the indications, evidences, and clinical trials. Onco Targets Ther. 2018;11:2865-2874. doi:10.2147/OTT.S164651

8. Zhang Z-M, Lai EC, Zhang C, et al. The strategies for treating primary hepatocellular carcinoma with portal vein tumor thrombus. Int J Surg. 2015;20:8-16.

9. Edeline J, Crouzet L, Campillo-Gimenez B, et al. Selective internal radiation therapy compared with sorafenib for hepatocellular carcinoma with portal vein thrombosis. Eur J Nucl Med Mol Imaging. 2016;43:635-643. doi:10.1007/s00259-015-3210-7

10. Lee IJ, Chung JW, Kim HC, et al. Extrahepatic collateral artery supply to the tumor thrombi of hepatocellular carcinoma invading inferior vena cava: the prevalence and determinant factors. $J$ Vasc Interv Radiol. 2009;20:22-29. doi:10.1016/j.jvir.2008.09.030

11. Rim CH, Jeong B-K, Kim TH, Hee Kim J, Kang H-C, Seong J. Effectiveness and feasibility of external beam radiotherapy for hepatocellular carcinoma with inferior vena cava and/or right atrium involvement: a multicenter trial in Korea (KROG 17-10). Int $J$ Radiat Biol. 2020;96:759-766. doi:10.1080/ 09553002.2020.1721607

12. Wang Y, Yuan L, Ge R-L, Sun Y, Wei G. Survival benefit of surgical treatment for hepatocellular carcinoma with inferior vena cava/right atrium tumor thrombus: results of a retrospective cohort study. Ann Surg Oncol. 2013;20:914-922. doi:10.1245/s10434-012-2646-2

13. Wakayama K, Kamiyama T, Yokoo H, et al. Surgical management of hepatocellular carcinoma with tumor thrombi in the inferior vena cava or right atrium. World J Surg Oncol. 2013;11:259. doi:10.1186/1477-7819-11-259 
14. Zeng Z-C, Fan J, Tang Z-Y, et al. A comparison of treatment combinations with and without radiotherapy for hepatocellular carcinoma with portal vein and/or inferior vena cava tumor thrombus. Int J Radiat Oncol Biol Phys. 2005;61:432-443. doi:10.1016/j. ijrobp.2004.05.025

15. Koo JE, Kim JH, Lim Y-S, et al. Combination of transarterial chemoembolization and three-dimensional conformal radiotherapy for hepatocellular carcinoma with inferior vena cava tumor thrombus. Int J Radiat Oncol Biol Phys. 2010;78:180-187. doi:10.1016/j. ijrobp.2009.07.1730

16. Llovet JM, Ricci S, Mazzaferro V, et al. Sorafenib in advanced hepatocellular carcinoma. $N$ Engl J Med. 2008;359:378-390. doi:10.1056/NEJMoa0708857

17. Lou J, Li Y, Liang K, et al. Hypofractionated radiotherapy as a salvage treatment for recurrent hepatocellular carcinoma with inferior vena cava/right atrium tumor thrombus: a multi-center analysis. BMC Cancer. 2019;19:668. doi:10.1186/s12885-019-5870-3

18. Tanaka Y, Nakazawa T, Komori S, et al. Radiotherapy for patients with unresectable advanced hepatocellular carcinoma with invasion to intrahepatic large vessels: efficacy and outcomes. $J$ Gastroenterol Hepatol. 2014;29:352-357. doi:10.1111/jgh.12333

19. Hanterdsith B. Fatal pulmonary thromboembolism due to inferior vena cava thrombosis. Ann Vasc Dis. 2011;4:121-123. doi:10.3400/ avd.cr. 10.00021

20. Goto H, Hashimoto M, Akamatsu D, et al. Surgical resection and inferior vena cava reconstruction for treatment of the malignant tumor: technical success and outcomes. Ann Vasc Dis. 2014;7:120-126. doi:10.3400/avd.oa.13-00125

21. Matsukuma S, Eguchi H, Wada H, et al. Liver resection with thrombectomy for patients with hepatocellular carcinoma and tumour thrombus in the inferior vena cava or right atrium. BJS Open. 2020;4:241. doi:10.1002/bjs5.50258

22. Kasai Y, Hatano E, Seo S, et al. Proposal of selection criteria for operative resection of hepatocellular carcinoma with inferior vena cava tumor thrombus incorporating hepatic arterial infusion chemotherapy. Surgery. 2017;162:742-751. doi:10.1016/j. surg.2017.05.011

23. Li Y, Liu F, Yang L, Meng Y, Li A, Pan M. External-beam radiation therapy versus surgery in the treatment of hepatocellular carcinoma with inferior vena cava/right atrium tumor thrombi. Asia Pacific $J$ Clin Oncol. 2019;15:316-322. doi:10.1111/ajco.13194

24. Komatsu S, Kido M, Asari S, et al. Particle radiotherapy, a novel external radiation therapy, versus liver resection for hepatocellular carcinoma accompanied with inferior vena cava tumor thrombus: a matched-pair analysis. Surgery. 2017;162:1241-1249. doi:10.1016/j. surg.2017.08.006

25. Lee HA, Park S, Seo YS, Yoon WS, Shin IS, Rim CH. Surgery versus external beam radiotherapy for hepatocellular carcinoma involving the inferior vena cava or right atrium: a systematic review and meta-analysis. J Hepatobiliary Pancreat Sci. 2020. doi:10.1002/ jhbp. 865

26. Galle PR, Forner A, Llovet JM; Liver EAFTSOT. EASL clinical practice guidelines: management of hepatocellular carcinoma. J Hepatol. 2018;69:182-236. doi:10.1016/j.jhep.2018.03.019

27. Chern M, Chuang V, Cheng T, Lin Z, Lin Y. Transcatheter arterial chemoembolization for advanced hepatocellular carcinoma with inferior vena cava and right atrial tumors. Cardiovasc Intervent Radiol. 2008;31:735-744. doi:10.1007/s00270-008-9342-4

28. Jeon SH, Park KS, Kim YH, et al. Incidence and risk factors of acute hepatic failure after transcatheter arterial chemoembolization for hepatocellular carcinoma. Korean J Gastroenterol. 2007;50:176-182.

29. Kokudo T, Hasegawa K, Yamamoto S, et al. Surgical treatment of hepatocellular carcinoma associated with hepatic vein tumor thrombosis. $J$ Hepatol. 2014;61:583-588. doi:10.1016/j. jhep.2014.04.032
30. Zhang XP, Liu YC, Chen ZH, et al. Postoperative adjuvant transarterial chemoembolization improves outcomes of hepatocellular carcinoma associated with hepatic vein invasion: a propensity score matching analysis. Ann Surg Oncol. 2019;26:1465-1473. doi:10.1245/s10434-019-07223-z

31. Lee HA, Lee YS, Kim BK, et al. Change in the recurrence pattern and predictors over time after complete cure of hepatocellular carcinoma. Gut Liver. 2020. doi:10.5009/gnl20101

32. Sumie S, Kuromatsu R, Okuda K, et al. Microvascular invasion in patients with hepatocellular carcinoma and its predictable clinicopathological factors. Ann Surg Oncol. 2008;15:1375-1382. doi:10.1245/s10434-008-9846-9

33. Chun JM, Kwon HJ, Sohn J, et al. Prognostic factors after early recurrence in patients who underwent curative resection for hepatocellular carcinoma. J Surg Oncol. 2011;103:148-151. doi:10.1002/ jso. 21786

34. Pao TH, Hsueh WT, Chang WL, et al. Radiotherapy for inferior vena cava tumor thrombus in patients with hepatocellular carcinoma. $B M C$ Cancer. 2019;19:560. doi:10.1186/s12885-019-5654-9

35. Tokuda K. Pathomorphological study on hepatocellular carcinoma. Kurume Med J. 1978;41:1044-1051.

36. Yoon JS, Lee HA, Park JY, et al. Hepatocellular carcinoma in Korea between 2008 and 2011: an analysis of Korean nationwide cancer registry. J Liver Cancer. 2020;20:41-52. doi:10.17998/jlc.20.1.41

37. Xia F, Wu -L-L, Lau W-Y, et al. Adjuvant sorafenib after heptectomy for Barcelona Clinic Liver Cancer-stage C hepatocellular carcinoma patients. World J Gastroenterol. 2016;22:5384. doi:10.3748/wjg.v22. i23.5384

38. Li J, Hou Y, Cai X-B, Liu B. Sorafenib after resection improves the outcome of BCLC stage C hepatocellular carcinoma. World $J$ Gastroenterol. 2016;22:4034. doi:10.3748/wjg.v22.i15.4034

39. Wang DX, Yang X, Lin JZ, et al. Efficacy and safety of lenvatinib for patients with advanced hepatocellular carcinoma: a retrospective, real-world study conducted in China. World $J$ Gastroenterol. 2020;26:4465-4478. doi:10.3748/wjg.v26.i30.4465

40. Wang Y, Deng W, Li N, et al. Combining immunotherapy and radiotherapy for cancer treatment: current challenges and future directions. Front Pharmacol. 2018;9:185. doi:10.3389/fphar.2018.00185

41. Kudo M, Finn RS, Qin S, et al. Lenvatinib versus sorafenib in first-line treatment of patients with unresectable hepatocellular carcinoma: a randomised phase 3 non-inferiority trial. Lancet. 2018;391:1163-1173. doi:10.1016/S0140-6736(18)30207-1

42. Van Limbergen EJ, De Ruysscher DK, Olivo Pimentel V, et al. Combining radiotherapy with immunotherapy: the past, the present and the future. $B r \quad J$ Radiol. 2017;90:20170157. doi:10.1259/ bjr.20170157

43. Demaria S, Kawashima N, Yang AM, et al. Immune-mediated inhibition of metastases after treatment with local radiation and CTLA-4 blockade in a mouse model of breast cancer. Clin Cancer Res. 2005;11:728-734

44. Zeng J, See AP, Phallen J, et al. Anti-PD-1 blockade and stereotactic radiation produce long-term survival in mice with intracranial gliomas. Int $J$ Radiat Oncol Biol Phys. 2013;86:343-349. doi:10.1016/j.ijrobp.2012.12.025

45. Sharabi AB, Nirschl CJ, Kochel CM, et al. Stereotactic radiation therapy augments antigen-specific PD-1-mediated antitumor immune responses via cross-presentation of tumor antigen. Cancer Immunol Res. 2015;3:345-355. doi:10.1158/2326-6066.CIR-14-0196

46. Dovedi SJ, Cheadle EJ, Popple AL, et al. Fractionated radiation therapy stimulates antitumor immunity mediated by both resident and infiltrating polyclonal T-cell populations when combined with PD-1 blockade. Clin Cancer Res. 2017;23:5514-5526. doi:10.1158/ 1078-0432.CCR-16-1673

47. Deng L, Liang $\mathrm{H}$, Burnette $\mathrm{B}$, et al. Irradiation and anti-PD-L1 treatment synergistically promote antitumor immunity in mice. J Clin Invest. 2014;124:687-695. doi:10.1172/JCI67313 
48. Choi C, Yoo GS, Cho WK, Park HC. Optimizing radiotherapy with immune checkpoint blockade in hepatocellular carcinoma. World J Gastroenterol. 2019;25:2416-2429. doi:10.3748/wjg.v25.i20.2416

49. Qin A, Rengan R, Lee S, et al. A pilot study of Atezolizumab plus hypofractionated image-guided radiotherapy for the treatment of advanced non-small cell lung cancer. Int J Radiat Oncol Biol Phys. 2019.

50. Wang SJ, Haffty B. Radiotherapy as a New Player in Immuno-Oncology. Cancers (Basel). 2018;10:515. doi:10.3390/ cancers 10120515

51. Kim KJ, Kim JH, Lee SJ, Lee EJ, Shin EC, Seong J. Radiation improves antitumor effect of immune checkpoint inhibitor in murine hepatocellular carcinoma model. Oncotarget. 2017;8:41242-41255. doi:10.18632/oncotarget.17168
52. Friedman D, Baird JR, Young KH, et al. Programmed cell death-1 blockade enhances response to stereotactic radiation in an orthotopic murine model of hepatocellular carcinoma. Hepatol Res. 2017;47:702-714. doi:10.1111/hepr.12789

53. Lee JH, Lee J-H, Lim Y-S, et al. Adjuvant immunotherapy with autologous cytokine-induced killer cells for hepatocellular carcinoma. Gastroenterology. 2015;148:1383-1391. e1386. doi:10.1053/j.gastro.2015.02.055

\section{Publish your work in this journal}

The Journal of Hepatocellular Carcinoma is an international, peerreviewed, open access journal that offers a platform for the dissemination and study of clinical, translational and basic research findings in this rapidly developing field. Development in areas including, but not limited to, epidemiology, vaccination, hepatitis therapy, pathology and molecular tumor classification and prognostication are all considered for publication. The manuscript management system is completely online and includes a very quick and fair peer-review system, which is all easy to use. Visit http://www.dovepress.com/ testimonials.php to read real quotes from published authors. 